Published in final edited form as:

Oncol Nurs Forum. 2016 September 01; 43(5): 625-635. doi:10.1188/16.ONF.625-635.

\title{
Cancer Journey for American Indians and Alaska Natives in the Pacific Northwest
}

\author{
Emily A. Haozous, PhD, RN, FAAN, \\ Associate professor in the College of Nursing at the University of New Mexico in Albuquerque \\ Ardith Doorenbos, PhD, RN, FAAN, \\ Professor in the School of Nursing and Medicine at the University of Washington in Seattle
}

Lori A. Alvord, MD,

Associate dean of Student Affairs and Admissions in the College of Medicine at the University of Arizona in Tucson

David R. Flum, MD, MPH, and

Professor in the School of Medicine at the University of Washington

Arden M. Morris, MD, MPH

Associate professor in the Medical School at Stanford University in California

\section{Abstract}

Purpose/Objectives-To describe the experiences of American Indian and Alaska Native cancer survivors to improve understanding of the trajectory of cancer treatment.

Research Approach-Qualitative focus group research.

Setting-Rural and geographically isolated American Indian and Alaska Native communities in the Pacific Northwest.

Participants-30 American Indian and Alaska Native cancer survivors or caregivers.

Methodologic Approach-The authors analyzed data from two focus groups with cancer survivors by using thematic analysis informed by indigenous methodologies.

Findings-Based on focus group findings, the authors developed a conceptual model of the cancer experience called Rough Waters. Participants described their cancer experience as a collective journey involving family and friends and requiring resources to offset challenges along the way. Dominant themes were delays, isolation, communication, money, advocacy, spirituality, and family involvement.

Conclusions-American Indians and Alaska Natives in the Pacific Northwest have special cultural needs during cancer care. The current study provides examples that can guide patientprovider interactions.

Haozous can be reached at ehaozous@salud.unm.edu, with copy to editor at ONFEditor@ons.org.

All authors contributed to the conceptualization and design, analysis, and manuscript preparation. Doorenbos completed the data collection. 
Interpretation-Using the metaphor of cancer as a journey, clinicians can begin a dialogue to identify what will impede or assist the cancer journey for their American Indian and Alaska Native patients.

\section{Keywords}

American Indian; Alaska Native; indigenous methodologies; qualitative; cancer survivorship

Although cancer incidence in American Indians and Alaska Natives (AIs/ANs) is lower nationwide than in the general population, mortality is disproportionately high (Holck, Day, \& Provost, 2013; Hoopes, Petersen, Vinson, \& Lopez, 2012). Regional variation (Espey et al., 2007; Hoopes et al., 2012; Wiggins et al., 2008) suggests that this high mortality is because of a constellation of nonclinical factors, including cultural, systemic, and economic barriers to care. Distance to oncology treatment centers, inability to pay for specialty care, high cost of transportation, lack of access to cancer screening, and distrust of healthcare providers all make oncology care difficult for AIs/ANs (Burhansstipanov \& Hollow, 2001; Guadagnolo et al., 2009; Haozous \& Knobf, 2013; Haozous, Knobf, \& Brant, 2011; Sequist et al., 2011).

Unfortunately, the barriers that obstruct access to care for AIs/ANs also act as barriers to research, such as perceived poor-quality communication between researchers and participants (Mead et al., 2013). As a result, little is known about their experience of cancer or their perceptions of cancer treatment. For example, a survey of AIs/ANs who underwent cancer screening revealed that they either knew little about their family history of cancer or declined to disclose it in a survey (Schumacher et al., 2008). The same study also found that relatively few $\mathrm{AI} / \mathrm{AN}$ women receive regular screening for cervical, breast, or colorectal cancer. However, these data were limited to ANs and southwestern AIs, with no AI/AN respondents in the Pacific Northwest (Schumacher et al., 2008). Another study, which described a telehealth project originating in the Seattle area and using a calendar mail-out to increase cancer screening, provides context for understanding the barriers to care (geographic and otherwise) described by AI/AN patients with cancer (Doorenbos, Demiris, et al., 2011; Doorenbos et al., 2010; Doorenbos, Jacobsen, Corpuz, Forquera, \& Buchwald, 2011). However, these studies offer little information on patient perspectives.

These studies clarify certain aspects of the cancer experience for AIs/ANs, but they do not address the full continuum of care. Such a gap in knowledge makes it difficult to assess community needs or to address the disproportionately high mortality suffered by AIs/ANs. Understanding the personal experience of cancer among AIs/ANs may help to inform interventions that improve care for cancer and other life-altering illnesses by improving communication and facilitating the delivery of culturally congruent care. For this reason, the research team determined that qualitative inquiry was necessary to build a foundational understanding of the experience of cancer treatment for AIs/ANs in the Pacific Northwest. This article investigates the experience of AI/AN cancer survivors in the region and offers an indigenous conceptual model. 


\section{Methodologic Approach}

The authors convened focus groups with AI/AN cancer survivors, family caregivers, and community leaders residing in the Pacific Northwest. The goal was to expand the understanding of the trajectory of cancer treatment for geographically isolated AI/AN communities in this region. All study procedures were presented before the tribal councils of the participating tribal groups for review and approval, and the University of Washington Human Subjects Division in Seattle reviewed the protocol prior to any research activities commenced. All participants provided verbal informed consent.

\section{Indigenous Methodologies}

The Western perspective on knowledge development has privileged a data-based, analytic, positivist epistemology (Campbell, 2014; Kovach, 2009; Newhouse, 2004; Simonds \& Christopher, 2013; Smith, 2012). This perspective has marginalized indigenous knowledge, which emerges from the intellectual mind, but is also engaged with body, spirit, history, dreams, collective knowledge, and knowledge of the natural world and its rhythms (Campbell, 2014; Newhouse, 2004). Indigenous methodologies, a research approach, emerged in response to the marginalization of Native science, recognizing that indigenous knowledge cannot exist in the Western research paradigm, where the context and relationships to the mind, body, spirit, and community are lost (Newhouse, 2004).

The authors' approach to data collection and analysis was guided by indigenous methodologies. Throughout the research process, the authors strove to prioritize the indigenous participants' and communities' ways of knowing and learning (Evans, Hole, Berg, Hutchinson, \& Sookraj, 2009; Louis, 2007; Smith, 2012). Although the tenets of indigenous methodologies vary from one study to another, investigators in this field generally agree that research with indigenous populations should be holistic, wellnessoriented, community-based, focused on indigenous knowledge, and designed for bidirectional learning (Evans et al., 2009; Louis, 2007).

The current study conformed to these guidelines by building knowledge about the cancer experience from the perspective of AI/AN participants. The authors interpreted focus group data with the goal of finding relevant language and imagery that resonated with AI/AN communities. The conduct of the focus groups placed the needs and priorities of the participants at the forefront. In the creation of the conceptual model, the authors proceeded with the enthusiastic feedback from the community as confirmation that the model was representative of the cancer experience for their community members, and permission was received to use this representation for research purposes. The authors received explicit confirmation through presentation of the model to the same tribal leader panel that originally gave permission to conduct this research.

\section{Participants}

The authors recruited 30 participants from two established cancer support groups, each located on a tribal reservation in the Pacific Northwest. This approach ensured that all participants in each group were familiar with and supportive of one another. The first focus 
group had 16 participants; the second had 14. Participants included people diagnosed with any type of cancer, as well as their family caregivers. The authors defined the sample size on the basis of findings that 30 participants is a sufficient size to achieve saturation in qualitative research that seeks to identify patterns and themes (Sandelowski, 1995). Given the small populations of the communities involved and the relatively small number of cancers diagnosed at any time, the names of the tribal communities and other demographic details are omitted from this report to protect participants' confidentiality.

\section{Data Collection and Analysis}

There were three phases to the process. Phase 1 included focus groups to identify constructs related to cancer care and treatment, phase 2 included taking the focus group results back to the focus group participant key informants to assess accuracy of the constructs, and phase 3 included conducting semistructured qualitative interviews with a subset of the original focus group participants. Phase 1 included two focus groups that were conducted with American Indian patients with cancer to understand major themes of their cancer surgical experience. Participants were tribal members who had been diagnosed with cancer and their family caregivers. Each focus group started with a dinner, an expected component of meetings in tribal communities. The focus group facilitator (AD) is well known in the community where the study was conducted. Although not AI or AN, the facilitator has been an active participant in community events and provides expert consultation to the tribes in this region. Each focus group was audio recorded and co-facilitated by a tribal elder who was also a cancer survivor. The mutual familiarity among participants and researchers was an important contributor to bidirectional learning because it helped reduce the mistrust that has historically prevented medical researchers from obtaining high-quality data in similar vulnerable populations (Guadagnolo et al., 2009).

A paid transcriptionist was used for the focus group transcripts. These transcripts were reviewed by the facilitators to verify accuracy, then the data were dei-dentified and analyzed to determine themes related to the cancer journey (Dixon-Woods, Agarwal, Jones, Young, \& Sutton, 2005; Sandelowski, 2000). The qualitative evaluation used content analysis and thematic description of focus group participants' discussion of the cancer journey to identify major topics within and across the different focus groups (Ayres, Kavanaugh, \& Knafl, 2003). Analysis involved a close reading of the transcripts, followed by in vivo coding of the transcripts. These in vivo codes were clustered into categories, and those developing categories were coded based on content. The categories were examined by the research team and grouped into clusters, which were analyzed to identify general themes (Hsieh \& Shannon, 2005). The emerging themes were discussed during research team meetings, and the team identified quotes exemplifying key themes. A preliminary model was developed from the themes. The preliminary model was a rough outline of a branching river, depicting the manner in which the cancer journey can be affected by advocacy or aggressive behavior.

Phase 2 key informant interviews drew from the same group of participants who were in the initial focus groups. Key informants were chosen because of their ability to articulate the cancer journey and having an understanding of cancer healthcare provision. Because of the small sizes of the communities involved and the relatively small numbers of cancers 
diagnosed at any time, the tribal communities and further demographic details are omitted from the current article to protect the confidentiality of the participants. Key informants from the participating tribal communities reviewed the preliminary results and made suggestions to the emerging themes, guiding the analysis process. This iterative process of tribal member input on the qualitative analysis interpretation led to a second round of analysis to confirm changes and a revision of the model from a river with two branches to one river without branches. The revised model integrated features from the natural world well known to tribes of the area, most notably a river made treacherous with rocks, waves, downed trees, and eddies, representing key themes from the data.

In phase 3, once key themes were established and agreed on by the research team, they were presented at a conference for $\mathrm{AI} / \mathrm{AN}$ cancer survivors, with more than 100 cancer survivors and their caregivers in attendance. The authors presented a sketch of the emerging conceptual model at this conference and received critical feedback on the accuracy and components of the conceptual model. Based on this feedback, the authors revised the model and asked an artist to render a visual image of the model, titled "Rough Waters" (see Figure 1). This model was presented to and approved by the appropriate tribal councils, and approval for dissemination was confirmed a second time.

Conceptual models drive new ideas by giving a foundation from which research can be built, providing direction and acting as a touchstone at critical decision-making points along the research path (Fawcett \& Gigliotti, 2001). They also create a tangible representation of the abstract concepts or actions that, when seen as a whole, respond to the need to define and elucidate the roles and actions of nursing as a holistic practice (Fawcett \& Gigliotti, 2001). The authors offer an emergent conceptual model that fulfills these important traditional purposes but is not constrained by the dominant cultural perception of relationships. In alignment with the indigenous methodologies approach, the conceptual model depicts the research results in a manner that is aesthetically, intellectually, and spiritually in alignment with the priority population.

\section{Findings}

Participants described cancer treatment as fraught with barriers, including treatment delays, financial challenges, problems with transportation, and poor communication with clinicians. They also identified important resources that alleviated some of these difficulties, including reliance on spirituality, family involvement, and advocacy by themselves and others. Based on the analysis of focus group discussions, the authors developed and validated a conceptual model of the cancer journey for people from these communities.

Because the participating tribes are from the Pacific Northwest, members of these tribes consider themselves water people, with the rhythm of their lives closely linked to seasonal activities that occur along the rivers and the ocean, as well as to the other living creatures that coexist around these waterways. For many coastal Pacific Northwest tribes, the spiritual center of the community is an annual canoe journey, a traditional practice that has been embraced with renewed passion in recent years (Paddle to Nisqually, 2016). 
The authors began with a first draft of the conceptual model that was drawn and modified on the basis of feedback from the research team. This illustration underwent multiple revisions in response to additional feedback from key informants and researchers. The first author created all preliminary drafts based on data analysis results, as well as team and informant feedback. Key informants clarified that they envisioned the cancer journey proceeding along one river without branching tributaries, leading to revisions and the generation of a nearfinal sketch. Once this version was approved, the concept was presented to an artist from the community for his rendition. The artist's version, Rough Waters, became the final image.

The authors chose to use imagery from nature in crafting the image. Like the annual canoe journey undertaken by Pacific Northwest tribes, the cancer journey may include quick and easily navigable routes, but travelers can often be swept into eddies and sandbars or endangered by rocks and other barriers along the way. The authors labeled these barriers "delays," "communication," "isolation," and "money." Participants asserted that the critical factors involved in easing their cancer journey were "advocacy," "spirituality," and "family involvement." The artist who painted the final image suggested that powerful animals from the geographic region be included parallel to the travelers in the canoe. These visual elements have a strong cultural resonance for the participants in the focus groups. Including them in the image makes it more meaningful from the AI/AN perspective, incorporating the hallmarks of indigenous methodologies and making it resonate for and from an indigenous perspective.

\section{Delays}

Participants noted several different types of delays along the cancer trajectory. For some, delays were a natural result of the fragmented healthcare system in which they received treatment. They described having to negotiate multiple systems to coordinate care. Little communication existed between the clinicians in these systems, creating extra delays in the crucial period before diagnosis and decision making about surgery. One participant described how he waited for a diagnosis and for someone on his healthcare team to take control of his care. In his view, diagnostic tests that seemed unnecessary were conducted because of delays and poor communication among providers. He said,

I have had five [colonoscopies] now, but [the physician] is slow to answer them, he is slow to give me an appointment and colonoscopy. They didn't want to do it at first.... So he's taking his time, and he sets an appointment up two weeks later, and he says, "In two weeks, I'm going to make a decision," and a month later, he says, "Well, I'm going to do another colonoscopy."

Other participants described distress resulting from the delays that they experienced while trying to manage their symptoms. Control of pain was particularly difficult, and delay in this area was a key example of the distress experienced by patients and families. The previously quoted participant experienced fragmented care that caused delays in symptom management after his surgery. He said,

So, I had stitches, and I had staples, and I had a port in. I couldn't move....

Everybody kept saying, "Call [insurance provider]"; [Insurance provider] says,

"Call your provider." And everybody was saying, "Call this person, this person," so 
I have four different providers who were taking care of me, and no one is really being responsible for my pain.

\section{Communication}

Communication was another dominant theme. Poor communication between family members, across medical teams, and between healthcare providers and patients all had a powerful negative effect on participants' experience of cancer, whereas good communication was a key factor in establishing trust between patients and their providers. Communication was described in several ways, including verbal, nonverbal, visual, and behavioral.

Most participants described feeling different from patients who were not AI/AN seen in their care settings. This feeling contributed to a sense of alienation that negatively affected their experience. One participant observed that she received more respectful care from healthcare professionals when she made an effort to dress up for her appointments. She said, "When I go to the hospital, I have to dress up because I notice, when I dress up, I'm treated differently... If I just go in in my blue jeans and T-shirt and my canoe sweatshirt ... it's immediately drugs." When she wore her everyday clothes, she was perceived as a drug user seeking pain medication, and when she wore nicer clothing, she received care that she considered more appropriate to her situation.

Participants also conveyed their understanding of what was expected of them as patients when they interacted with clinicians who were not AI or AN. They understood that they were expected to ask questions despite that inquisitive behavior was perceived as being aggressive. This type of questioning of healthcare providers that is commonly expected in a typical medical encounter was inconsistent with their culture. One participant described an experience in which her son was receiving difficult information about his cancer diagnosis at a time when she was not able to be at his side to assist with communication. She said,

I think that the surgeon had gone in and told [my son], and, knowing how our people are, I just think that he probably didn't ask any questions. You know, he was probably told, "You have colon cancer of this kind, and we're going to do this and this and this," and probably [he] won't say anything, so maybe the doctors think that we understand. I just think that people don't ask questions.

Participants were aware that some members of their community were apt to be stoic and that information would be difficult to illicit from people who do not express their emotions or complain about their pain. One participant said, "Our son was so stoic; we didn't even know he had cancer. This time of the year, he was out messing with his nets ... and his cancer, or whatever it was, it burst while he was out there." Participants expected their healthcare providers to give them information in words that made sense to them and matched their health literacy level. Confidence with health literacy ranged widely among the participants. One participant, who indicated high confidence in her health literacy, noted that she was able to ask questions in a clear and concise manner when her presurgical information included diagrams and technical language referring to the procedure.

Similar to the woman who felt she needed to dress up for medical visits, other participants stated that they were treated "differently" by their healthcare providers and that this 
difference reduced their ability to trust providers. They saw this instance of mistrust as part of a long history of suspicion among AIs/ANs for representatives of the majority culture. They described a process within their own culture by which trust is earned by outsiders, and they extended this process to healthcare providers. One participant said,

I remember growing up and my parents teaching me [that] you don't just jump out and say something to people. You check them out before you do that. That was very hard for me when I went to college to break that barrier because I ... didn't trust anybody, very untrusting.

Communication emerged as a key strategy for establishing trust, but few participants had stories of trusting relationships with clinicians. They were best able to describe mistrust through stories of clinical exchanges in which their providers had spoken inappropriately. One participant said,

It was a non-Indian that did it the first time, he blurted out, I had malignant meningi- ... malignant something. And then I went home, and I didn't have a clue what I had.... So I thought there was a real big difference for being Native American.

Participants also recounted situations in which providers' actions betrayed their trust. Such situations made them feel that their own care was less important to providers than that of other patients. One participant was pregnant at the time of her cancer diagnosis, but her pregnancy was not detected until after she recovered from cancer surgery, and she was forced to make the difficult decision to terminate the pregnancy. She said,

I think the hospital could have done one or two steps. They didn't give me a urine sample, and [I came] to find out four months later I was pregnant when I went through the surgery.... I was getting ready to do my radiation, and the radiologist found out I was four months pregnant. They just didn't do that one extra step, and I think they do it to everyone else.

Participants who reported having an advocate were also the most likely to report trusting relationships. Those patients felt they had the benefit of a team to help navigate through their cancer journey. Having a trusting relationship with someone in the healthcare setting depended strongly on communication, and communication was built on mutual trust.

\section{Isolation}

Participants repeatedly emphasized that understanding the AI/AN way of life is important for anyone outside of those communities who wishes to interact. However, they found that their healthcare providers had no such understanding and no apparent interest in trying to understand. As one participant expressed,

They don't understand us. Our culture is so different. They don't understand the Native American way of life, the way they eat, the way they were born. It still affects, it is going to affect probably until, the rest of the time being.

According to participants, if clinicians made even a small effort to understand the AI/AN cultures and lifestyles, they would be taking a big step toward establishing trust. Apart from 
any philosophical issues, participants noted that the logistics of care could be improved if providers acquired the most basic understanding of cultural issues. For example, many participants described the importance of having their family and friends around them at times of illness for practical, emotional, and spiritual support. However, they found that their providers who were not AI or AN were unwilling to create new policies within healthcare institutions to permit frequent or multiple visitors. One participant said,

What they don't understand is that, all these people wanted to come in and visit me $\ldots$ and they tell you no. But I tell you what, my family came in.... When they wanted them to leave, they're not going to leave. That is when the care starts getting really bad because we're there with our family and our friends, and they want to shut them out and not inform them of ... what is happening, and that is all what our religious and cultural belief is.

\section{Money}

Finances and access to resources were another pervasive theme. Although AIs/ANs in the communities studied have private health care, money and access to resources were frequently cited as challenges. Some participants spoke of being denied care for lack of payment. Others who had good health insurance coverage still had trouble obtaining payment for services. Many also cited transportation costs as an issue and frequently mentioned the use of an existing program that provided prepaid gas credit cards for transportation to cancer treatment appointments. Participants noted that the combined costs of cancer care are extremely high, including transportation, food, lodging, and family needs during treatment. They regarded being away from home for treatment as a particular burden that created additional worries for patients and their families. In a population with limited resources, these financial concerns had the potential to be catastrophic. One participant said,

The gas cards that the program gives us, those are a big help because, with a lot of the tests that I have to do, I have to cleanse my body out for a colonoscopy. That costs $\$ 29$ [for] medication, and then you have to have the drinks. You can't eat, stuff like that, so you're spending all that money just to clean your body out for that one test, and the gas money to go to chemotherapy, the doctor's appointments ... because I would go there at $8 \mathrm{am}$, stay there until $12 \mathrm{pm}$, come back, go back Wednesday, get my port taken out, so it wasn't just one day. It was two days, and those gift cards ... helped out a lot.

For this participant, having assistance to cover these relatively small expenses made an enormous difference in his cancer experience.

Participants observed that ability to pay was linked to quality of care. Patients who lacked private insurance and, therefore, had to rely on the Indian Health Service (IHS) Contract Health Services for payment observed a marked difference in the care they received. They attributed this difference to the slow repayment process associated with IHS. As one participant described, individuals who had private health insurance had access to a range of healthcare providers that were not available to those who relied on IHS for healthcare needs. In addition, participants perceived that the IHS system gave worse care. A participant said, 
[My sister] was the one out there on the harbor [in the community]. She got the worst care, but, once they discovered what kind of insurance she had, then they gave her a different doctor, so that was the biggest question out there, was, "What kind of insurance do you have?" If it's IHS, some of them don't even want to see you because the tribes, they are so slow on their payment process.

\section{Advocacy}

Advocacy was another recurrent theme. Participants stressed the importance of advocacy from multiple levels, including advocating for themselves, having family members advocate on their behalf, and having a clinician advocate for them. They also emphasized the need to learn about their treatment and use that knowledge in their advocacy. One participant said,

I think, as long as family members go to the doctor appointments, are visible when people come in to help, nurses or whoever, and are very vocal and question doctors if need be, you get better care. And, unfortunately, it should not be like that because a lot of people in our community that have cancer aren't that aggressive; people and their families aren't that aggressive.

Participants described the strategies they used to advocate on behalf of family members who were living with cancer. One participant said,

My gram had cancer and, from there, there have just been different people in our family that have had cancer. And one of the things I've noticed is that, if you're poor, you don't get very good care. We've all been poor, but one of the things that happens is, normally, when we have someone in the hospital, the family goes in to ensure that things are being done.

In discussing advocacy, participants often noted that they had to be aggressive. The use of this term surprised the research team. On further consultation with key informants, the researchers learned that aggressive behavior is contrary to social and cultural norms in their community. Therefore, advocating for family members put informants outside of their comfort zone at a time when they were already likely to be socially and personally compromised by the experience of cancer.

\section{Spirituality}

Participants in both focus groups described strong ties to the Indian Shaker Church religious tradition. This Western-infused religious sect was one component of a dual spiritual path. Dual spirituality of this type (indigenous plus Western) is found in many AI/AN communities. For the current study population, spirituality and religiosity were also tied to family and lifestyle. In a follow-up discussion, participants reinforced this theme, agreeing that spirituality was a key component of their culture. Prayer and religion were important coping mechanisms for these participants. A family member of a patient with cancer said, "But what do you say to a person that has just been told that their husband has cancer? So of course we prayed. My upbringing was to pray, and so ... she got comforted." Hospital systems did not always understand or support the spirituality endorsed by participants. Another participant said, 
But with traditional beliefs and understandings, hospital and aftercare people in the hospital don't understand it and do not know how to acknowledge our faith, our fears, or anything with that.... Even when you're continually going, like I am, there isn't anybody that takes time to understand; they look at you like you're still in the woods.

Participants also reported that their healthcare providers ridiculed the rituals that were associated with prayer, whether these rituals were from an indigenous or Indian Shaker Church tradition. A participant said,

Our prayers and some of our spiritual ways are different from the White society.... People used to come in the hospital to pray for some of the people. [Providers] would poke fun and say, "Well, whatever, you know, they're going to ring a bell for you, and it's going to heal you." It was very racist.

\section{Family Involvement}

Cancer researchers have long recognized that cancer is not an individual experience, but one that affects family and friends, as well as the patient. Focus group participants reinforced this understanding, asserting that cancer is a collective journey. Although the patient is the natural center of this narrative, the family provides context and support for the patient. Participants stressed that a strong family presence was critical to creating a positive cancer trajectory. In the conceptual model, the family helps the patient navigate the cancer journey.

Family members provided practical support by assisting with activities of daily living. For example, family who lived near cancer care facilities could provide a place to stay for patients during treatment. Participants stressed the importance of including family members in patient education because the extended family network was closely involved with patient care. One participant said,

The aftercare for my sisters - there was none. I had to learn, me and one other sister had to learn how to take care of both of them, administer their medication. There was no public health nurse or anyone that was willing to show us how to do it. It was all liquid form, and we had to inject it in these IV bags. And we didn't have anyone at the hospital that was willing to show us how to do that. Instead, my sisters had to wait almost two weeks for someone to come out from Port Angeles and show us how to care for them because they were in between. They made too much for a home nurse to come out, but yet they didn't make enough to pay someone to come take care of them, and so that was the biggest problem both my sisters had.... We had to learn how to take care of them.

Family members described caregiver fatigue but were careful to protect patients from witnessing their distress. They relied on other family members, as well as nurses, to offset the burden of caring. One family member described caring for her dying father and the importance of a visiting nurse.

She was a very good nurse, and she did a good job. She would come sit with him, so I could have a break. You know [how] you get so into it because you're with them 24 hours a day taking care of them? Sometimes, I'd be so depressed and 
upset, I'd go out on the back porch and scream, let some of that frustration out. But she was a very good nurse, and she really helped us, like you've got family.

The nurse described in the excerpt was so important to the care of the speaker's father that she became "like family" at this critical time in their lives.

Participants also described family members as critical to cancer care. They reported encountering difficulties when family members were hospitalized because hospital regulations limited the number of visitors and the length of their visits. For the informants, having family members at the bedside at all times was an important part of patient care. One participant said,

They didn't understand why we stayed at the hospital the entire time our sisters were there, and we never left. And that was probably one of the biggest questions they would ask us, "What are you guys still doing here?" Well, my sisters are still here, that is what we're still doing here. We didn't care if we had to sleep in the waiting area.

Although participants described lives rich in social connections, they also felt that living the cancer journey could be isolating. In particular, they were concerned that obtaining care required time away from their families and tribal community. They were also concerned that insufficient services were available for cancer survivors who needed food and social support outside the home. They strongly believed that the home was a private place and that privacy needed to be protected. Although caregiving involves helping people with the activities of daily living, participants preferred social programs that operated outside the home to preserve privacy. They also voiced concerns about maintaining a hygienic home environment to protect immunocompromised patients from infection. By limiting visitors and caregiving as much as possible to external locations, the safety of the home was protected.

\section{Implications for Nursing}

Although the model created on the basis of the results is particularly relevant for the seafaring tribes of the Pacific Northwest, the metaphor of the journey resonates for many cultures. In clinical practice, providers who care for AIs/ANs might find this conceptual model useful for identifying barriers and promoters of progress along the cancer journey. The current authors have used this conceptual model as a tool to guide conversations with $\mathrm{AI} / \mathrm{AN}$ patients, asking them where they are on the journey, so the authors can work with them to determine where they need assistance to surmount barriers to care. In generating a model that has personal and cultural applicability for an indigenous audience, the authors hope that this dialogue can aid in addressing some of the cultural and systemic barriers between healthcare providers and their AI/AN patients, which may ultimately affect cancer mortality.

Culturally sensitive care also includes knowing about community resources that can ease a patient's cancer journey. For example, patients in the current study expected their families to be involved in all aspects of their health care, and many of them needed financial assistance with transportation. Understanding these needs is essential for delivering culturally 
congruent care in this population. One use of the results would be to create a toolkit for providers to facilitate culturally sensitive communication or identify community or local resources.

\section{Conclusion}

When compared against the literature describing the barriers and facilitators to cancer care in other marginalized and vulnerable communities, these results had several commonalities with the existing body of research. Poor communication between patients and healthcare providers is a common barrier to care, observed across the cancer spectrum and including African Americans, immigrant and migrant communities, and other minority groups (Bickell \& Cohen, 2008; Gany et al., 2013; Masi, Blackman, \& Peek, 2007). Difficulties accessing care is also an issue for African American and Afro-Caribbean men seeking prostate cancer screening, linking systemic barriers to lower screening, which has direct implications for patient outcomes (Lee, Consedine, Gonzalez, \& Spencer, 2012). Similar to the current authors' results, financial challenges are a clear concern for other minority groups, observed in general and in relation to insurance status in particular (Bickell \& Cohen, 2008; Lee et al., 2012; Masi et al., 2007). Access to reliable transportation has been observed as a clear barrier to cancer care that contributes to health disparities for racial and ethnic minority people with cancer (Murphy, Tseng, \& Shah, 2010). As was observed with the participants in this research, patient assistance programs are helpful in addressing concerns, such as transportation and childcare issues, that can hinder access to cancer treatment (Bickell \& Cohen, 2008). Unlike the current research, stigma and shame did not emerge as themes within these communities, but they have been observed in other AI/AN cultural groups, as well as other racial groups (Haozous et al., 2011; Lee-Lin, Menon, Nail, \& Lutz, 2012).

The conceptual model depicts the experience of cancer for AI/AN people in the Pacific Northwest. Participants described their cancer journey as a challenge that required stepping outside cultural norms. They had to face adversities associated with cancer and cancer treatment with aggressive determination, combined with the deep spiritual connection that is traditional in their culture. Participants in this research provided important information on the special cultural needs involved in medical care for AI/AN patients with cancer in the Pacific Northwest. They understood the experience of cancer as a collective journey involving family and friends, one that required special resources to offset the challenge of traveling between rural villages and urban treatment centers. They helped the researchers recognize that, although no one can predict which barriers might arise during the cancer journey, healthcare providers can still anticipate possible problems while working to reinforce the known strengths of family, spirituality, and advocacy in this patient population.

Practitioners who are not accustomed to working with AI/AN communities may be unfamiliar with the historic background of AI/AN spirituality. Many AIs/ANs in this region of the Pacific Northwest are members of the Indian Shaker Church, a Christian religion with indigenous origins, unrelated to the historic Shaker Church that emerged from the Quaker religion in 18th-century England (Neylan, 2011). The Indian Shaker Church is unique among Christian religions in that it integrates AI/AN spiritual practices with Christian practices in the religious rituals of ceremony (Struthers \& Eschiti, 2005). Participants in this 
study described their religion and more traditional, indigenous prayers as essential components of healing within a dual spiritual practice and are, in some circumstances, inseparable. The practice of combining indigenous spiritual practices, which are unrelated to Christianity, with Western-infused religious traditions is not uncommon in AI/AN communities. Dual spirituality denotes a spectrum of belief in many Native communities, such that some community members will adhere only to their indigenous spiritual practices, others will adhere only to Western spirituality, and others will combine the two in varying proportions (Burhansstipanov \& Hollow, 2001).

Creating a visual model was a key outcome of the use of indigenous methodologies. The authors wanted the results to resonate with the core values of community partners, a goal that is integral to indigenous approaches (Martin \& Mirraboopa, 2003). Although some research products may have importance on a national or international scale, the authors centered the present work on the needs, priorities, and worldview of the target population. The image has strong symbolic significance for the study community, offering concepts and language that can help patients and providers better understand the cancer journey. The authors expect that many AIs/ANs living with cancer will find it useful in conveying their cancer experience.

The authors recognize that the conceptual model is limited by its tailoring for AI/AN communities who have an orientation to the patterns of the coastal regions. However, the conceptual model and the process by which the authors found words and images to express the model provide an example of indigenous methodologies and subsequent results.

The study was also limited by the lack of demographic data that could place the results in a socioeconomic context. The primary focus was to understand the experience and perceptions of AI/AN patients with cancer. Because the data were collected in a population that is historically averse to research, the authors opted to limit the amount of personal demographic data that were requested from participants. The rationale was to avoid any offense that might interfere with elicitation of the primary study data.

Using indigenous methodologies to analyze and present the research findings, the authors developed a conceptual model that provides important insights into the cultural experience of cancer for AIs/ANs in two Pacific Northwest communities. The conceptual model that was developed from this research replaces the expected boxes and arrows of the Western design with a more holistic design that relies on the natural flow of water to indicate directionality and the inherent indigenous knowledge of the AI/AN participants within the target population to aid in interpretation. As with traditional Western conceptual models, this model has applicability in future research and current applicability in clinical practice. This research can help providers and AI/AN patients identify barriers and promoters in cancer care, as well as their effect on health outcomes. It can also help in designing interventions to address gaps in care, and it can facilitate a dialogue between community members and academic partners on the use of patient-centered research and the translation of such research to indigenous communities. 


\section{Acknowledgments}

The authors gratefully acknowledge the Collaborative to Improve Native Cancer Outcomes, including Dedra Buchwald, MD, David R. Flum, MD, MPH, Eva M. Garroutte, PhD, Angela A. Gonzales, PhD, Jeff A. Henderson, MD, MPH, Patricia N. Henderson, MD, MPH, Donald L. Patrick, PhD, MSPH, Shin-Ping Tu, MD, MPH, FACP, and Rachel L. Winer, PhD, for their editorial review, as well as Bob Haozous for creating and allowing the use of the artwork used in the Rough Waters model.

This research was funded by the National Institute of Health under Award Number P50CA148110.

\section{References}

Ayres L, Kavanaugh K, Knafl KA. Within-case and across-case approaches to qualitative data analysis. Qualitative Health Research. 2003; 13:871-883. DOI: 10.1177/1049732303013006008 [PubMed: 12891720]

Bickell NA, Cohen A. Understanding reasons for underuse: An approach to improve quality and reduce disparities in breast cancer treatment. Mount Sinai Journal of Medicine, New York. 2008; 75:23-30. DOI: $10.1002 / \mathrm{msj} .20013$

Burhansstipanov L, Hollow W. Native American cultural aspects of oncology nursing care. Seminars in Oncology Nursing. 2001; 17:206-219. DOI: 10.1053/sonu.2001.25950 [PubMed: 11523487]

Campbell TD. A clash of paradigms? Western and indigenous views on health research involving aboriginal peoples. Nurse Researcher. 2014; 21(6):39-43. DOI: 10.7748/nr.21.6.39.e1253

Dixon-Woods M, Agarwal S, Jones D, Young B, Sutton A. Synthesising qualitative and quantitative evidence: A review of possible methods. Journal of Health Services Research and Policy. 2005; 10:45-53. DOI: 10.1258/1355819052801804 [PubMed: 15667704]

Doorenbos AZ, Demiris G, Towle C, Kundu A, Revels L, Colven R, Buchwald D. Developing the native people for cancer control telehealth network. Telemedicine Journal and e-Health: The Official Journal of the American Telemedicine Association. 2011; 17:30-34. DOI: 10.1089/tmj.2010.0101 [PubMed: 21214371]

Doorenbos AZ, Eaton LH, Haozous E, Towle C, Revels L, Buchwald D. Satisfaction with telehealth for cancer support groups in rural American Indian and Alaska Native communities. Clinical Journal of Oncology Nursing. 2010; 14:765-770. DOI: 10.1188/10.CJON.765-770 [PubMed: 21112853]

Doorenbos AZ, Jacobsen C, Corpuz R, Forquera R, Buchwald D. A randomized controlled calendar mail-out to increase cancer screening among urban American Indian and Alaska Native patients. Journal of Cancer Education. 2011; 26:549-554. DOI: 10.1007/s13187-011-0217-z [PubMed: 21472495]

Espey DK, Wu XC, Swan J, Wiggins C, Jim MA, Ward E, Edwards BK. Annual report to the nation on the status of cancer, 1975-2004, featuring cancer in American Indians and Alaska Natives. Cancer. 2007; 110:2119-2152. DOI: 10.1002/cncr.23044 [PubMed: 17939129]

Evans M, Hole R, Berg LD, Hutchinson P, Sookraj D. Common insights, differing methodologies: Toward a fusion of indigenous methodologies, participatory action research, and white studies in an urban aboriginal research agenda. Qualitative Inquiry. 2009; 15:893-910. DOI: $10.1177 / 1077800409333392$

Fawcett J, Gigliotti E. Using conceptual models of nursing to guide nursing research: The case of the Neuman systems model. Nursing Science Quarterly. 2001; 14:339-345. DOI: 10.1177/089431840101400411 [PubMed: 11873373]

Gany F, Yogendran L, Massie D, Ramirez J, Lee T, Winkel G, Leng J. "Doctor, what do I have?" Knowledge of cancer diagnosis among immigrant/migrant minorities. Journal of Cancer Education. 2013; 28:165-170. [PubMed: 23093484]

Guadagnolo BA, Cina K, Helbig P, Molloy K, Reiner M, Cook EF, Petereit DG. Medical mistrust and less satisfaction with health care among Native Americans presenting for cancer treatment. Journal of Health Care for the Poor and Underserved. 2009; 20:210-226. [PubMed: 19202258] 
Haozous EA, Knobf MT. “All my tears were gone": Suffering and cancer pain in Southwest American Indians. Journal of Pain and Symptom Management. 2013; 45:1050-1060. DOI: 10.1016/ j.jpainsymman.2012.06.001 [PubMed: 22940564]

Haozous EA, Knobf MT, Brant JM. Understanding the cancer pain experience in American Indians of the northern plains. Psycho-Oncology. 2011; 20:404-410. DOI: 10.1002/pon.1741 [PubMed: 20878836]

Holck P, Day GE, Provost E. Mortality trends among Alaska Native people: Successes and challenges. International Journal of Circumpolar Health. 2013; 72doi: 10.3402/ijch.v72i0.21185

Hoopes MJ, Petersen P, Vinson E, Lopez K. Regional differences and tribal use of American Indian/ Alaska Native cancer data in the Pacific Northwest. Journal of Cancer Education. 2012; 27(Suppl):S73-S79. DOI: 10.1007/s13187-012-0325-4 [PubMed: 22281722]

Hsieh HF, Shannon SE. Three approaches to qualitative content analysis. Qualitative Health Research. 2005; 15:1277-1288. [PubMed: 16204405]

Kovach, M. Indigenous methodologies: Characteristics, conversations and contexts. Toronto, Canada: University of Toronto Press; 2009.

Lee DJ, Consedine NS, Gonzalez JR, Spencer BA. Association of healthcare barriers with prostatespecific antigen screening among African-American and Afro-Caribbean men. Urology. 2012; 80:556-563. DOI: 10.1016/j.urology.2012.02.085 [PubMed: 22789295]

Lee-Lin F, Menon U, Nail L, Lutz KF. Findings from focus groups indicating what Chinese American immigrant women think about breast cancer and breast cancer screening. Journal of Obstetric, Gynecologic, and Neonatal Nursing. 2012; 41:627-637.

Louis RP. Can you hear us now? Voices from the margin: Using indigenous methodologies in geographic research. Geographical Research. 2007; 45:130-139. DOI: 10.1111/j. 1745-5871.2007.00443.x

Martin KL, Mirraboopa B. Ways of knowing, being and doing: A theoretical framework and methods for indigenous and indigenist research. Journal of Australian Studies. 2003; 27:203-214. DOI: $10.1080 / 14443050309387838$

Masi CM, Blackman DJ, Peek ME. Interventions to enhance breast cancer screening, diagnosis, and treatment among racial and ethnic minority women. Medical Care Research and Review. 2007; 64(Suppl):195S-242S. DOI: 10.1177/1077558707305410 [PubMed: 17881627]

Mead EL, Doorenbos AZ, Javid SH, Haozous EA, Alvord LA, Flum DR, Morris AM. Shared decision-making for cancer care among racial and ethnic minorities: A systematic review. American Journal of Public Health. 2013; 103:e15-e29. DOI: 10.2105/ajph.2013.301631

Murphy MM, Tseng JF, Shah SA. Disparities in cancer care: An operative perspective. Surgery. 2010; 147:733-737. DOI: 10.1016/j.surg.2009.10.050 [PubMed: 19962161]

Newhouse D. Indigenous knowledge in a multicultural world. Native Studies Review. 2004; 15:139_ 154.

Neylan S. Shaking up Christianity: The Indian Shaker Church in the Canada-US Pacific Northwest. Journal of Religion. 2011; 91:188-222. DOI: 10.1086/658108

Paddle to Nisqually. Don't forget the water. 2016. Retrieved from http://www.paddletonisqually.com

Sandelowski M. Sample size in qualitative research. Research in Nursing and Health. 1995; 18:179_ 183. [PubMed: 7899572]

Sandelowski M. Whatever happened to qualitative description? Research in Nursing and Health. 2000; 23:334-340. DOI: 10.1002/1098-240X(200008)23:4<334::AID-NUR9>3.0.CO;2-G [PubMed: 10940958]

Schumacher MC, Slattery ML, Lanier AP, Ma KN, Edwards S, Ferucci ED, Tom-Orme L. Prevalence and predictors of cancer screening among American Indian and Alaska Native people: The EARTH study. Cancer Causes and Control. 2008; 19:725-737. DOI: 10.1007/s10552-008-9135-8 [PubMed: 18307048]

Sequist TD, Cullen T, Bernard K, Shaykevich S, Orav EJ, Ayanian JZ. Trends in quality of care and barriers to improvement in the Indian Health Service. Journal of General Internal Medicine. 2011; 26:480-486. DOI: 10.1007/s11606-010-1594-4 [PubMed: 21132462] 
Simonds VW, Christopher S. Adapting Western research methods to indigenous ways of knowing. American Journal of Public Health. 2013; 103:2185-2192. DOI: 10.2105/ajph.2012.301157 [PubMed: 23678897]

Smith, LT. Decolonizing methodologies: Research and indigenous peoples. 2nd. London, England: Zed Books; 2012.

Struthers R, Eschiti VS. Being healed by an indigenous traditional healer: Sacred healing stories of Native Americans. Part II. Complementary Therapies in Clinical Practice. 2005; 11:78-86. DOI: 10.1016/j.ctnm.2004.05.002 [PubMed: 16028347]

Wiggins CL, Espey DK, Wingo PA, Kaur JS, Wilson RT, Swan J, Lanier AP. Cancer among American Indians and Alaska Natives in the United States, 1999-2004. Cancer. 2008; 113(Suppl):11421152. DOI: 10.1002/cncr.23734 [PubMed: 18720375] 


\section{Knowledge Translation}

- This model helps providers and American Indian and Alaska Native patients identify barriers and promoters in cancer care, as well as their effect on health outcomes.

- Clinicians can design interventions to address gaps in care based on the model.

- This research can facilitate a dialogue between community members and academic partners on the use of patient-centered research and the translation of that research to indigenous communities. 


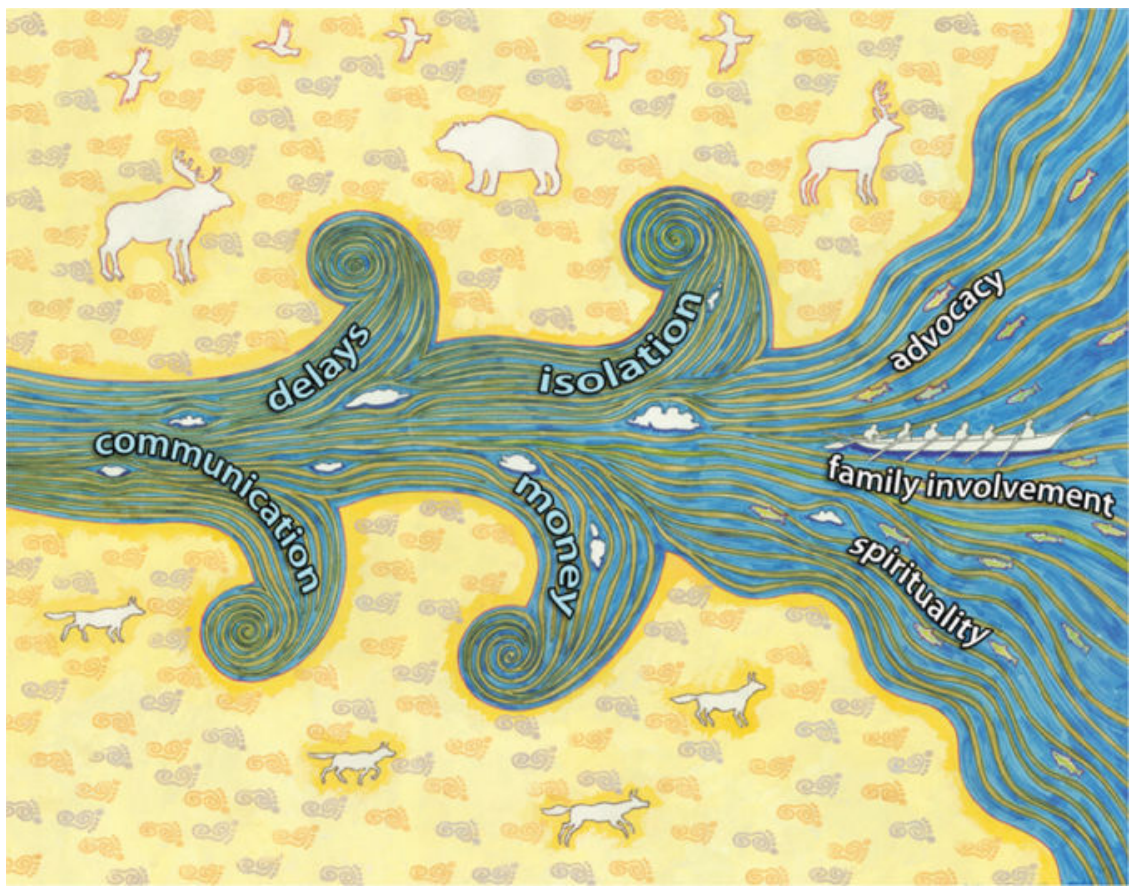

FIGURE 1. Rough Waters Conceptual Model

Note. Image courtesy of Bob Haozous. Used with permission. 